\title{
Simulation and Analysis on Signal Acquisition of BDS Receiver with The Aid of INS
}

\author{
Shulei Chen, Xiaqing Tang, Xuwei Cheng, Junqiang Gao and Zepeng Sun \\ Department of Control Engineering, Academy of Armored Force Engineering, Beijing 100072, China
}

\begin{abstract}
Improving the dynamic performances of BDS receiver with INS-aided is the hotspot of the current navigation research, and the key problem is how to improve the performance of signal acquisition. This paper has some research and analyses about the theory and mathematic model of signal acquisition. Though the simulation of vehicle trajectory, the author acquired the results of signal acquisition performance of BDS receiver with the aid of INS composing of $10 \mathrm{deg} / \mathrm{h}$ bias drift gyros and $100 \mathrm{ug}$ bias drift accelerometer. The experiment results illustrate that the average signal acquisition time of BDS receiver with the low accuracy INS aided is shortened greatly, the searching scope of Doppler frequency shift and code phase are narrowed, thus the signal acquisition performance of BDS receiver is improved. This research can serve as good instruction for the development of integrated navigation.
\end{abstract}

Keywords-BDS; signal acquisition; INS-aided; performance analysis

\section{INTRODUCTION}

As the continuous improvement of science and technology, information war has become the mainstream in military, the army needs the navigation instrument with high accuracy sensor. Therefore the startup-time of navigation system must be shorter and signal acquisition and tracking must be quick. With the rapid development of navigation technology, we usually use INS aided satellite receiver to improve its performance in harsh environment. This paper uses traditional serial acquisition method based on time domain to acquire Beidou satellite signal , then analyzes the performance indexes of the BDS receiver. What's more, The author use MATLAB simulate the course of signal acquisition with the aid of low precision INS though simulating the vehicle trajectory of the carrier. In the end, this paper has some comparison and analysis of the results of signal acquisition with or without INS-aided. The work has the guiding significance for BDS/INS integrated system design.

\section{THE THEORY OF SIGNAL ACQUISITION WITH THE AID OF} INS

\section{A. The Serial Acquisition Scheme Based On Gliding Correlation}

As for the satellites of BDS, they have the same carrier frequency and transmitting time of the signal at the beginning. The carrier frequency of signal will be changed along with the motion of the satellite and receiver (Doppler effect), what' more, the code phase of carrier will be changed too. So we acquire signal by determining the carrier frequency and code phase. There are many methods of signal acquisition, the simplest one is the serial acquisition scheme based on gliding correlation. The satellite signal become IF signal though the receiver RF, the RF outputs IF signal to acquisition module after sampling. The carrier genetator and pseudo code generator of receiver generate signal which frequency and code phase could be changed, then get a result after handling the IF signal and local signal by modulation integral. If the result is greater than the fixed threshold, signal acquisition is success. If not, we must change the frequency and code phase of local signal and repeat the previous step. [1]Figure 1 shows signal acquisition structure model.

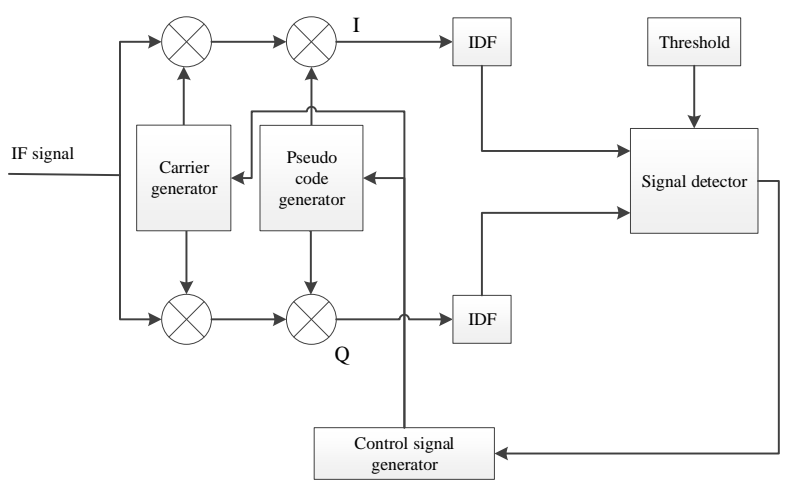

FIGURE I. THE SIGNAL ACQUISITION MODEL

The signal becomes IF signal though RF front. And the IF signal can be obtained as:

$$
S_{I F}(t)=\sqrt{2 P_{S}} C(t-\tau) \mathrm{D}(\mathrm{t}-\tau) \cos \left[\omega_{I F} t+\phi(t)\right]+n(t)
$$

Where, $P_{\mathrm{S}}$ is power of IF signal; $C(t)$ is pseudo code; $\tau$ is time delay; $\mathrm{D}(\mathrm{t})$ is navigation message; $\omega_{I F}$ is frequency of IF signal; $\phi(t)$ is initial code phase; $n(t)$ is white noise.

Two ways of orthogonal signals produced by local generators and are multiplied with input signals through mixer I and Q, and the model is as follows:

$$
I(t)=s_{I F}(t) \sqrt{2} \cos \left[\left(\omega_{I F}+\Delta \hat{\omega}\right) t+\widehat{\phi}_{0}\right]
$$




$$
Q(t)=s_{I F}(t) \sqrt{2} \sin \left[\left(\omega_{I F}+\Delta \widehat{\omega}\right) t+\widehat{\phi}_{0}\right]
$$

The multiplying unit can be regarded as a low pass filter due to the integrator behind it. Therefore, the high-frequency in the above formula can be ignored, which has been simplified as follows (noise is neglected):

$$
\bar{I}_{P}=D \sqrt{P_{S}} \int_{0}^{T 1} C(t-\tau) C(t-\widehat{\tau}) \cos \left[\Delta \hat{\omega} t+\delta \widehat{\phi}_{0}\right] d t+\bar{N}_{I}
$$

$$
\bar{Q}_{P}=D \sqrt{P_{S}} \int_{0}^{T 1} C(t-\tau) C(t-\widehat{\tau}) \sin \left[\Delta \widehat{\omega} t+\delta \widehat{\phi}_{0}\right] d t+\bar{N}_{Q}
$$

Select the modulus from the results of the above two formulas and make threshold judgment. If the value of the result high enough, the signal can be acquired. The coordinates of the peak correspond to the carrier frequency and code phase of the signal. Otherwise, adjust the local carrier frequency and code phase by the control device until the presence of peak exceeding the detection threshold . [2]

\section{B. Signal Acquisition With Ins-Aided}

In order to acquire satellite signals rapidly when it is started, BDS receiver is required to cut down the search scope for satellite signals. Meanwhile, three kinds of information are necessary to identify the search scope, including satellite ephemeris, the speed and position of the receiver as well as the current BDS time. If INS navigation parts can assist inputting navigation information such as Doppler frequency shift information and code phase information into the satellite receiver in advance, it will greatly shorten the search scope above the Doppler frequency domain. With the same step size, the smaller the search scope is, the less time will be spent. In such circumstances, the TTFF (Time To First Fix) will also be reduced. [3]

When the satellite receiver receives the satellite signals, the Doppler frequency shift of carrier can be represented in the following:

$$
f= \pm f_{s}+f_{r e v} \pm \Delta f_{s}+\Delta f_{r e v}
$$

Where, $f_{s}$ is the Doppler frequency shift arising from the movement of the satellite. $f_{\text {rev }}$ is the Doppler frequency shift caused by the movement of the carrier. $\Delta f_{s}$ is to the frequency drift due to the clock errors of BDS satellite while $\Delta f_{\text {rev }}$ is the frequency drift produced by the clock errors of satellite receiver. Since the minimum errors of satellite clocks can be ignored, the formula can be simplified as:

$$
f \approx f_{r e v} \pm f_{s}+\Delta f_{r e v}
$$

And the mathematic model of Doppler frequency shift is:

$$
f_{b} \approx \frac{\left(V_{r e v}-V_{s}\right) \vec{e}}{c}
$$

Where, $V_{\text {rev }}$ and $V_{s}$ respectively represent the satellite receiver and the speed of the satellite. $\vec{e}$ is the unit direction vector. Supported by INS and ephemeris almanac, we can know about the satellite and the speed of the receiver in real time. Furthermore, we can figure out the value for $f_{\text {rev }}$ and $f_{s}$, subsequently shortening the Doppler search scope. If the speed of the satellite provided by the satellite ephemeris is accurate, the performance of INS will directly affect the acquisition performance of the satellite receiver. We will make the study from the perspective of satellite receiver's performance index to analyze the influences of INS on the receiver in multiple ways.

\section{PERFORMANCE INDEXES OF SATELLITE RECEIVER}

\section{A. Detection Probability Analysis}

Detection probability and false alarm probability are two important indicators to detect the performance of the satellite receiver. Both of these indicators determine the reliability of the signal acquisition. Larger detection probability and smaller false alarm probability will lead to more reliable acquisition. Under the condition of non-coherent integration, we adopt the simulation of detection probability to reflect the acquisition performance of the receiver with or without assistance. The detection value of non-coherent integration is:

$$
Z=\frac{1}{K} \sum_{K=1}^{K} \sqrt{I_{K}^{2}+Q_{K}^{2}}
$$

Figure out the squares of the ambiguity functions for $I$ and $Q$ within $\mathrm{K}$ periods and add them together from the counterpoints to obtain the final detection value. [4]

Define two assumptions:

$\mathrm{H} 0$ : assume the presence of noise $(\mathrm{A}=0)$ without signal

$\mathrm{H} 1$ : assume the presence of signal $(\mathrm{A}>0)$

When $\mathrm{A}=0$, the detection quantity $\mathrm{Z}$ is distributed as Rayleigh, and the false alarm probability is:

$$
P_{f a}=\int_{V_{t}}^{\infty} p\left(z \mid H_{0}\right) \mathrm{dz}=\mathrm{e}^{-\frac{V_{t}^{2}}{2 \sigma_{n}^{2}}}
$$

Where, $V_{t}$ is the detection threshold value and $\sigma_{n}^{2}$ is the noise power. We can obtain that the detection threshold value is: 


$$
V_{t}=\sigma_{n} \sqrt{-2 \ln P_{f a}}
$$

If the value of false alarm probability $P_{f a}$ is obtained, the detection threshold value of the system can be figured out.

If $\mathrm{H} 1$ is valid, the detection probability can be expressed as follows:

$$
P_{d}(B)=Q_{1}\left(\frac{\sqrt{\lambda}}{\sigma_{n}}, \frac{\sqrt{\beta}}{\sigma_{n}}\right) \approx \mathrm{Q}_{1}\left(\frac{\sqrt{C N}}{N_{0} f_{s}}, \frac{\sqrt{N \beta}}{N_{0} f_{s}}\right)
$$

$\mathrm{Q}_{1}(\cdot, \cdot)$ is first-order Marcum'Q-Function, which is:

$$
\mathrm{Q}_{K}(\mathrm{a}, \mathrm{b})=\frac{1}{a^{K-1}} \int_{b}^{+\infty} x^{K} e^{-\left(\frac{x^{2}+a^{2}}{2}\right)} I_{K-1}(\mathrm{ax}) \mathrm{dx}
$$

In the above formula, $I_{K-1}$ is the first class Bessel function of K-1 order. For easy calculation, the detection probability can be expressed as follows:

$$
P_{D}(\mathrm{~B})=\frac{1-\left[1-e^{-\left(\frac{B}{2 \sigma_{n}^{2}}\right.}\right]^{N}}{N \cdot e^{-\left(\frac{B}{2 \sigma_{n}^{2}}\right)}} \cdot Q_{1}\left(\frac{A}{\sigma_{n}}, \frac{\sqrt{B}}{\sigma_{n}}\right)
$$

With the help of the software simulation, we can work out a relationship graph illustrating the changes of detection probabilities for different Doppler errors as the carrier-to-noise ratios in cases where the false alarm probability is 0.001 , the search step size of code phase is 0.5 chip and the pre-detection integration is $2 \mathrm{~ms}$.

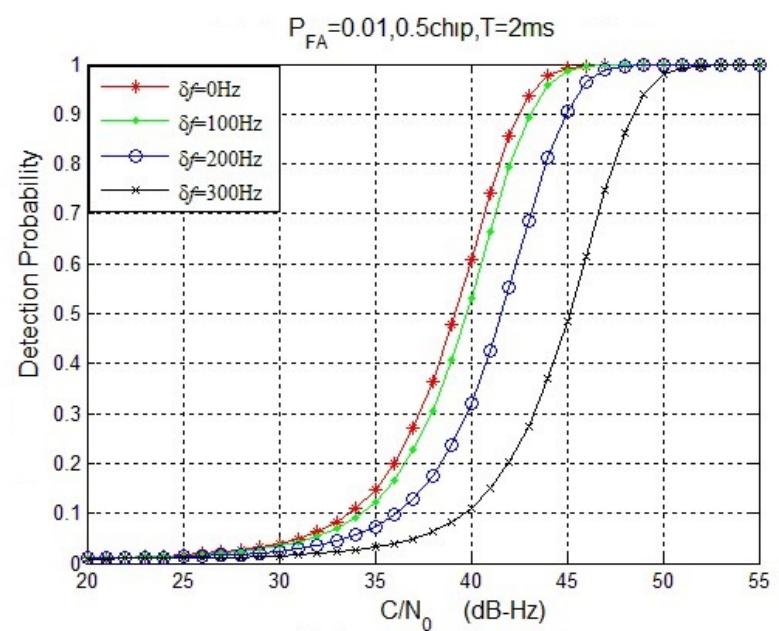

FIGURE II. RELATIONSHIP GRAPH ILLUSTRATING THE CHANGES OF DETECTION PROBABILITIES FOR DIFFERENT DOPPLER ERRORS AS THE CARRIER-TO-NOISE RATIOS
As shown in the figure, when the Doppler error is less than $100 \mathrm{~Hz}$, it exerts the minimum influence on the detection probability.

\section{B. Calculation Analysis}

With the serial acquisition algorithm based on time domain, assume $f_{\text {unc }}$ and $t_{\text {unc }}$ respectively refer to the uncertain section scopes searched by frequency and code, $f_{\text {bin }}$ and $t_{\text {bin }}$ respectively represent the frequency search step size and code search step size[5], then the total search cell is:

The receiver always processes discrete data in practical operation. $I(\tau)$ and $\mathrm{Q}(\tau)$ can be discretized as $I(k)$ and $Q(k)$. With $\mathrm{k}=0,1, \ldots, \mathrm{n}-1$, if we adopt regular serial acquisition method, every change in the value of $\mathrm{k}$ would require $\mathrm{n}$ times of multiplication and addition operations. Because there are $n$ values of $k, n 2$ times of multiplication and addition operations are required in a carrier frequency. For $\mathbf{M}$ times of frequency searches, Mn2 times of calculations are needed. Thus, we can make calculation analysis based on the changes of the acquisition scope.

\section{Average Acquisition Time Analysis}

The average residence time for each cell is:

$$
T_{d w e l l}=\left(\frac{2-P_{d}}{P_{d}}\right)\left(\mathrm{k}_{p} P_{f a}+1\right) T
$$

Where, ${ }{ }_{d}$ is detection probability, the loss factor of $\mathrm{k}_{p}$ is 1, $P_{f a}$ is false alarm probability, and $\mathrm{T}$ is integral time, the required acquisition time is:

$$
T=T_{d w e l l} \frac{2 f_{u n c} t_{u n c}}{f_{b i n} t_{b i n}}
$$

\section{SIMULATION EXPERIMENT}

This experiment has adopted MATLAB software to simulate the motion curve of the vehicle with low-precision INS navigation equipment constituted by 100ug precision accelerometer and 1deg/h precision gyroscope. The curve starts from the five-pointed star, moving toward the north at first. It returns to the initial position after 600s. The x-axis is latitude line and the $y$-axis is the longitude line. The initial speed is 0 , the maximum acceleration is $10 \mathrm{~m} / \mathrm{s} 2$ and the maximum angular velocity is $30 \mathrm{deg} / \mathrm{s}$. The motion curve is shown in Figure 3 as follows: 


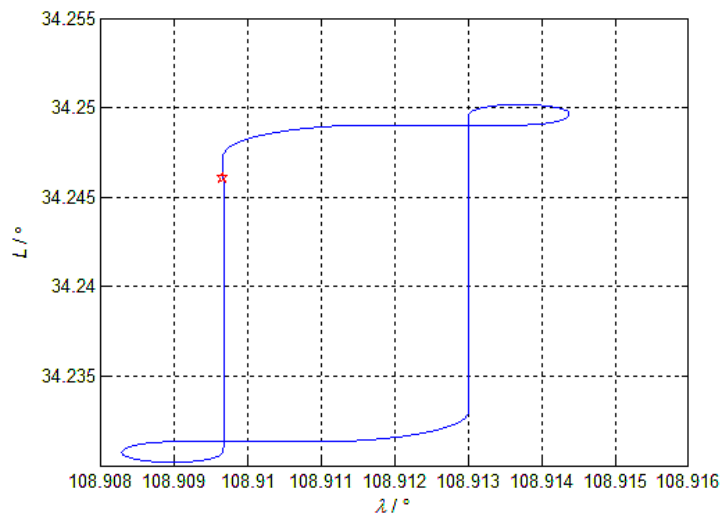

FIGURE III. THE SIMULATION OF VEHICLE TRAJECTORY

The speed error and position error in the northeast calculated by low-precision inertial navigation within 600s are respectively shown in Figure 4 and Figure 5. Within 600s, the northern speed error does not exceed $4 \mathrm{~m} / \mathrm{s}$ and the eastern speed error is no more than $3 \mathrm{~m} / \mathrm{s}$. Since it is a route test on the simulated floor, the speed error is nearly close to zero. The variance of Doppler frequency shift can also be calculated by INS speed error. While the latitude error reaches up to 350s and longitude error approaches to 250s within 600s. The analysis on the detection probability of acquisition supported by INS from the perspectives of Doppler error and code phase is shown as next chapter.

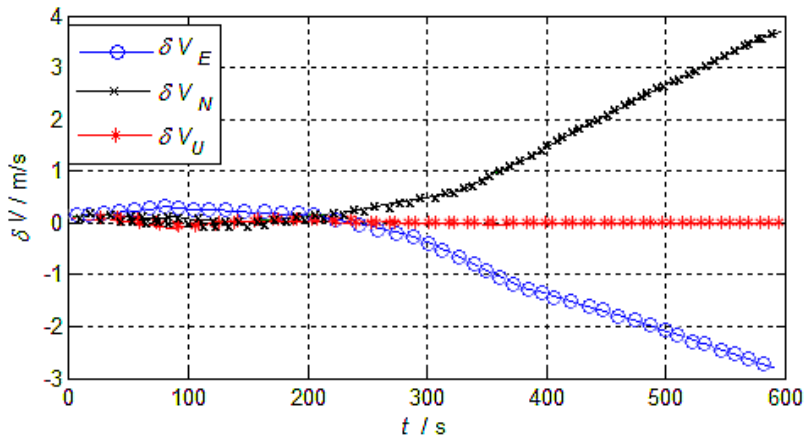

FIGURE IV. SPEED ERROR OF INS

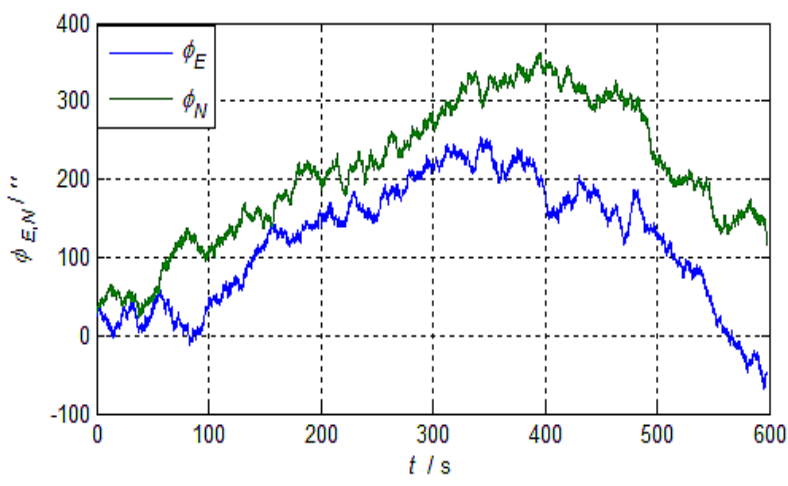

FIGURE V. LONGITUDE AND LATITUDE ERRORS OF INS

\section{Result ANALysis}

Apply the speed and position information of INS as a result of 300s' operation to assist BDS in capturing the satellite signal. After analyzing the acquisition probability before and after the assistance, we make the graph in Figure 6. The sensitivity of its signal acquisition has been improved by 4-5dB-Hz.

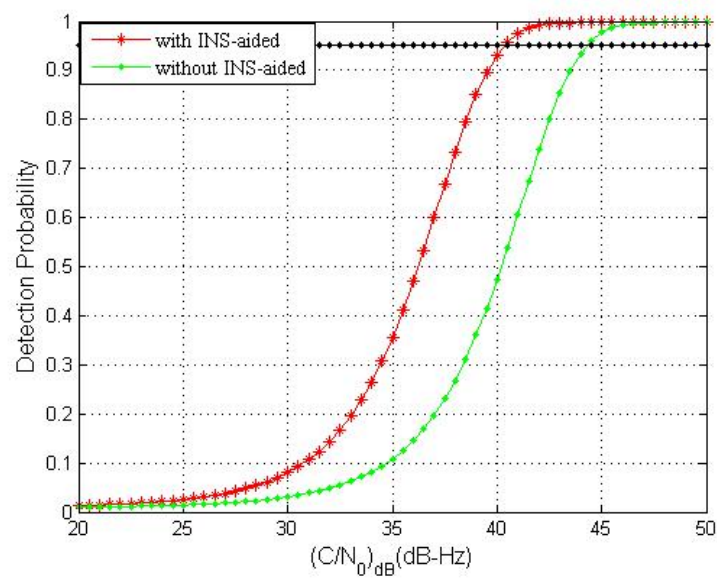

FIGURE VI. COMPARISON GRAPH ABOUT THE ACQUISITION SENSITIVITY BEFORE AND AFTER THE INS-AIDED

If the receiver is supported by low-precision INS calculating information and ephemeris, the search scope of Doppler frequency shift can be declined to $[-150 \mathrm{kHz},+150 \mathrm{kHz}]$ in a short time, which is less than the step size of the frequency search. In conclusion, the table which has illustrated the calculation quantity with/without INS assistance is listed in Table 1 as follows:

TABLE I. CALCULATION QUANTITY WITH/WITHOUT INS-AIDED

\begin{tabular}{|c|c|c|c|c|}
\hline $\begin{array}{c}\text { Way of } \\
\text { Acquisiti } \\
\text { on }\end{array}$ & Assistance & Search scope & $\begin{array}{c}\text { Number } \\
\text { of search } \\
\text { cells }\end{array}$ & $\begin{array}{c}\text { Calculation } \\
\text { quantity }\end{array}$ \\
\hline $\begin{array}{c}\text { Serial } \\
\text { acquisitio } \\
\text { n }\end{array}$ & No & {$[10 \mathrm{kHz},+10 \mathrm{kHz}]$} & 163680 & 669778560 \\
\hline $\begin{array}{c}\text { Serial } \\
\text { acquisitio } \\
\text { n }\end{array}$ & Yes & {$[150 \mathrm{~Hz},+150 \mathrm{~Hz}]$} & 4092 & 16744464 \\
\hline
\end{tabular}

Obtained from the above table, with the help of INS and ephemeris, the calculation quantity and energy will be declined by an order of magnitudes.

Given that the detection probability of BDS receiver is 0.95 , false alarm probability is 0.01 , the integral time of time domain acquisition is $2 \mathrm{~ms}$, and the system clock of receiver is $5.714 \mathrm{MHz}$, the required time without INS assistance can be figured out as:

$$
T=T_{d w e l l} \frac{2 f_{u n c} t_{u n c}}{f_{\text {bin }} t_{b i n}}=362 \mathrm{~s}
$$

The required time with INS assistance can be figured out 


$$
T_{\text {INS }}=T_{d w e l l} \frac{2 f_{\text {unc }} t_{\text {unc }}}{f_{\text {bin }} t_{\text {bin }}}=9 \mathrm{~s}
$$

Therefore, even with low-precision INS-aided, the acquisition time of satellite signal can be considerably reduced.

\section{CONCLUSION}

This paper have shown that even the low-precision INS could effectively improve the acquisition sensitivity of the receiver, which would greatly lower the calculation quantity and improve the acquisition time of the receiver. The research achievements made by this article can instruct the design of the INS/BDS combined navigation from the perspective of acquisition signal, which can help with signal track of receiver and the further study on the filter technology as well as provide vital practical significances to the information war.

\section{REFERENCES}

[1] Zhang G L, Zeng J. Principle and Technology of Integrated Navigation.Xi'an: Xi'an Jiantong University Press,2008,pp,31-46.

[2] Lashley M, Bevly D M. Performance Comparison of Deep Integration and Tight Coupling[J]. Navigation, 2013, 60(3),pp, 159-178.

[3] Liu J Y, Zeng Q H. Navigation System Theory and Application. Xi'an: Nwupress,2010,pp,152-154.

[4] Lu Y.BDS/GPS Dual Mode Software Reiver. Beijing : Electronic Industry Press, 2016,pp,134-136.

[5] He X F. Performance analysis on high dynamic signal acquisition aided by SINS for GNSS satellites. Journal of Chinese Inertial Technology, 2011,19 (4) ,pp,446-451. 